Impacto turístico que genera la Marca País "Ecuador ama la vida"

\title{
Impacto turístico que genera la Marca País "Ecuador ama la vida"
}

\section{Impact of tourism generated by the Country Brand "Ecuador loves life"}

\section{Impacto turístico que gera a Marca País "Equador ama la vida"}

Luci C. Salas-Narváez I

luci.salasn@ug.edu.ec

Alexis H. Villacís-Aveiga ${ }^{\text {II }}$

washington.villacisav@ug.edu.ec

Recibido: 02 de septiembre de 2017 * Corregido: 11 de noviembre de 2017 * Aceptado: 26 de diciembre de 2017

I. Magister en Administración de Empresas Mención en Recursos Humanos y Marketing, Profesora de Segunda Enseñanza Especialización: Mercadotecnia y Publicidad, Licenciada en Ciencias de la Educación Especialización: Mercadotecnia y Publicidad, Docente de la Universidad de Guayaquil, Guayaquil, Guayas, Ecuador.

II. Master of Science in Agricultural Economics, Ingeniero en Administración de Agronegocios, Docente de la Universidad de Guayaquil, Guayaquil, Guayas, Ecuador. 
Dom. Cien., ISSN: 2477-8818

Vol. 4, núm. 1, enero, 2018, pp. 603-618

http://dominiodelasciencias.com/ojs/index.php/es/index 


\title{
Resumen
}

El desarrollo de una marca que representa a un país, se considera como una estrategia conservadora de una imagen estable ante el mercado internacional; además de ello, lo más importante, es una forma de fomentar en los ciudadanos el consumo de los productos hechos en sus tierras, empezando por ellos mismos. El presente artículo tiene como objetivo realizar un análisis, revisión y recopilación de información con respecto al desarrollo turístico, que se da en sector costa de la República del Ecuador, con relación al impulso de la Marca País, bajo su lema "Ecuador ama la vida". Por lo tanto, fue necesario analizar teóricamente los temas relacionados y de esta manera llegar a conclusiones que permitan conocer la relación que estos términos tienen entre sí, como la evolución del sector turístico en los últimos tiempos. La revisión de la información que se realiza en este artículo, parte de la necesidad que se tiene, de dar a conocer la terminología aplicada a este tipo de fenómenos; partiendo de un análisis crítico hasta llegar a las más acertadas conclusiones posibles en base a referentes empíricos.

Palabras clave: planificación turística; marketing turístico; marca país; patrimonio cultural.

\begin{abstract}
The development of a brand that represents a country, is considered as a conservative strategy of a stable image before the international market; moreover, what is most important is a way of encouraging citizens to consume products made on their land, starting with themselves. The objective of this article is to analyze, review and compile information regarding tourism development in the coastal sector of the Republic of Ecuador, in relation to the promotion of the Country Brand, under its motto "Ecuador loves life ". Therefore, it was necessary to analyze theoretically the related issues and in this way reach conclusions that allow knowing the relationship that these terms have with each other, such as the evolution of the tourism sector in recent times. The revision of the information that is made in this article, part of the necessity that has, to give to know the terminology applied to this type of phenomena; starting from a critical analysis until arriving at the most successful possible conclusions based on empirical referents.
\end{abstract}

Keywords: tourism planning; tourism marketing; brand country; cultural heritage. 


\section{Resumo}

O desenvolvimento de uma marca que representa um país, se considera como uma estratégia conservadora de uma imagem estabelecida no mercado internacional; Além disso, mais importante, é uma forma de fomentar nos cidadãos do consumo dos produtos em sus tierras, começando por eles mesmos. O presente artigo tem como objetivo realizar um relatório, revisão e recopilação de informação sobre o desenvolvimento turístico, que se da no setor Costa da República do Equador, com relação ao impulso da Marca País, sob o lema "Equador ama la vida ". Por lo tanto, foi necessário analizar teóricamente os temas relacionados e desta forma chegar a conclusões que permitam conhecer a relação que estes bens têm entre sí, como a evolução do setor turístico nos últimos tempos. A revisão da informação que se realiza no presente artigo, parte dos pedidos que tem tem de definir a terminologia aplicada a este tipo de fenómenos; Participe de um relatório crítico para chegar a las mais acertadas conclusões possíveis em base a referentes empíricos.

Palavras chave: turística; marketing turístico; marca país; patrimonio cultural.

\section{Introducción}

En los últimos años el sector del Turismo se ha convertido en uno de los segmentos económicos que poco a poco se ha ido desarrollando en todo el planeta, a través de derivados como alojamiento, gastronomía entre otros, ha ido demostrado que es un sector con cifras de ganancias e inversión nunca vistas en esta área. (Marzano, 2013). Cabe indicar, que compañías, negocios y empresas dedicadas a este rubro se han expandido notablemente en los diferentes países del globo; siendo así Ecuador uno de ellos.

Por lo que, al ser un mercado en expansión, en donde la competencia está empezando a ser cada vez más directa y fuerte, ha motivado a que el marketing se vea involucrado en la gestión de estrategias y planes que están concentrados en mejorar la situación de las empresas hoteleras o turísticas, generando un nuevo concepto que se ha venido desarrollando en los últimos tiempos, englobando los servicios, infraestructura y demás elementos que puedan representar, en si todo lo que de cierta forma una nación tiene para dar. La Marca País, este es el término novedoso, ha sido ampliamente empleado en otros países del globo terráqueo, el mismo no siempre está relacionado con el sector turístico.

La Marca País, está ligado a la nación, siendo ejemplo de calidad, eficiencia, entre otros; teniendo como idea lograr gran acogida en varios países, con este aspecto se pretende impulsar al país como 
Impacto turístico que genera la Marca País "Ecuador ama la vida"

una marca ante el consumidor, haciendo uso de diversos medios entre los que se incluyen: la publicidad local e internacional, así como inculcar valores claves para este tipo de actividades, como por ejemplo la amabilidad o cordialidad, entre la población en general. Sin embargo e independientemente del desarrollo de la Marca País como tal, se debe recordar que si esta u otras estrategias se pretenden ejecutar orientados al sector turístico, se necesita estar al tanto de todos los factores que puedan de alguna forma $u$ otra, influir en esta actividad considerando que es un tipo de mercado distinto, que se ve afectado por elementos que no suelen ser los mismos que influyen a otros servicios, ypor lo tanto se requiere formular estrategias especializadas en este sector de la economía.

Estrategias que potencien el conocimiento sobre la diversidad de climas y regiones, la multiculturalidad, la biodiversidad y el patrimonio histórico, cultural y artístico, la atención al turista y el respaldo de un ambiente en general óptimo (altas medidas de seguridad, situación social y política estable) son esenciales para un desarrollo positivo de estas actividades. En el caso concreto de nuestro turismo, este ha crecido enormemente. (Costa \& G. Bosovsky, 2012). Ecuador no es el primer país en adoptar una marca para sí mismo, y se ha unido en la tendencia que impulsa vender naciones con un objetivo claro, el reconocimiento y la diferenciación. Cabe destacar el gran repunte que ha tenido el Turismo en otros países, que generalmente no habían sido considerados como los más visitados, entre los que destacamos el caso de Costa Rica, Perú, y también nuestro país, Ecuador.

Es así, como mediante el análisis de las diferentes estrategias de marketing y de la publicidad vinculadas al desarrollo de esta táctica en particular, se espera entender la manera cómo estas actividades han logrado orientarse al sector turístico, e incentivar su expansión y su desarrollo en la región litoral de nuestro país, pudiendo emitir un juicio objetivo sobre qué tan beneficioso ha sido hasta ahora la creación de esa identidad vinculada a la Marca Ecuador.

\section{Metodología}

Se usará el método analítico tomando como punto de partida el conocimiento buscado, y se retrocede, suponiendo órdenes de dependencia en los que ese conocimiento está incluido, hasta lograr la conexión con cadenas deductivas que son ya conocidas y evidentes. (Caimi, 2003, pág. 39) 
Impacto turístico que genera la Marca País "Ecuador ama la vida"

El método utilizado ayudó al procesamiento de la información, la cual ha sido recopilada durante la investigación, con aquello se pretende extraer elementos teóricos y referentes empíricos útiles para la fundamentación del trabajo.

El método deductivo también se utilizó, ya que "Es un método de razonamiento que consiste en tomar conclusiones generales para explicaciones particulares". (Bernal, 2006, pág. 56). Este será empleado en la elaboración de las conclusiones, las cuales servirán para presentar los resultados del trabajo de investigación.

\section{Desarrollo}

El Turismo ha experimentado un crecimiento considerable en los últimos tiempos, volcando los esfuerzos por incentivar esta lucrativa actividad con el fin de optimar la situación económica del país y mejorar la calidad de vida de sus habitantes. (Ministerio de Turismo del Ecuador, 2012); aspectos en expansión del sector terciario o de los servicios en las economías en vías de desarrollo, como el crecimiento del sector turístico ya que, después de todo, el turismo es en sí mismo es un servicio que se puede ofrecer a quien lo necesite, está destinado a satisfacer una necesidad existente en el mercado, como cualquier otro servicio, y por lo tanto requiere de planificación y de estudios previos para poder asegurar que se tendrá éxito (Bejarano, 2012).

Por ello, el área correspondiente al Turismo necesita de un enfoque que emplee similares herramientas, que ya se utilizan en otros sectores económicos pero sin dejar de lado las particularidades que tienen este tipo de servicios, con el fin de lograr un posicionamiento notable, tanto en los mercados locales como en los internacionales, pues "el turismo de hoy en día ha abierto paso al fortalecimiento de nuevas formas más complejas y perfeccionadas, para los nuevos turistas la parte fundamental de viajar hacia un destino cualquiera, se basa en las experiencias culturales que puedan tener" (Talavera, 2003).

$\mathrm{Y}$ es que, el turismo se adapta a las nuevas demandas en el que se incluye nuevas formas turísticas con el fin de satisfacer dichas experiencias. Estas actividades reflejadas principalmente en la imagen hacen alusión a una mayor posibilidad de gasto, "en general y sobre todo referido a los clientes interesados por lo rural y lo cultural, la demanda se centra en lo que las personas hacen" (Keller, 2012). 
Impacto turístico que genera la Marca País "Ecuador ama la vida"

Por lo que, en los últimos años existe una agresiva campaña publicitaria, que pretende ofrecer los atractivos que el país posee, por ejemplo, el Ecuador ofrece una amplia gama de destinos y productos turísticos en los Andes, la Costa y la Amazonía, donde la mayoría de los productos se relacionan con la naturaleza como el ecoturismo, turismo de aventura y agroturismo (Castellanos, 2011).

Cabe destacar que la mejor forma de que los turistas conozcan y respeten el patrimonio del lugar que visitan, es que, de alguna manera, se sientan identificados.

Como ya se ha mencionado anteriormente, el turismo es uno de los sectores que más se ha desarrollado en los últimos años en el Ecuador, alcanzando una participación significativa en el PIB, este crecimiento ha venido acompañado de una competencia cada vez más grande y feroz entre los diferentes países ofertantes para poder lograr una participación mayor en este mercado en expansión. (Ministerio de Turismo del Ecuador, 2013). Estos mayores niveles de competencia existentes entre los destinos turísticos han provocado que muchos en este sector necesiten tener un conocimiento más amplio tanto de los movimientos de los usuarios y como también de las diferentes fases del desarrollo de mercados, para de esta manera alcanzar un mayor grado de especialización y de técnica a la hora de proveer los servicios turísticos (Cantallops, 2013).

Sin embargo, el sector turístico suele estar influenciado por elementos que no siempre están presentes en otro tipo de empresas de servicios. El atractivo de los lugares o destinos turísticos puede variar según la época en que nos encontremos, debido a variables tales como el clima, la fecha de los ciclos vacacionales, etc., o la realización de eventos especiales que se promocionen como imán, para que los potenciales turistas lleguen a la localidad, se hospeden y gasten en la ciudad sin importar que sea una época poco común para eso. (Amaral Moretti \& Lenzi, 2013). Y es que, la aplicación de métodos tradicionales del Marketing, a este tipo de servicios puede por lo tanto ser útil en distintos grados. Así "El desarrollo del Marketing Mix para los destinos dependerá de su naturaleza, su mercado objetivo, y el entorno que lo rodea" (Bigne, Font, \& Andreu, 2014).

Es decir que los distintos sectores poblacionales a los que se apunte, y las diferentes circunstancias en las que se desenvuelva el destino turístico deben siempre ser tomadas en cuenta antes de desarrollar cualquier tipo de planificación de marketing, relacionado con potencializar el destino en cuestión y beneficios y servicios que este ofrezca. 
Impacto turístico que genera la Marca País "Ecuador ama la vida"

Sin embargo, hay que siempre mantener en cuenta que el trato a los clientes debe ser el mejor, se debe vender la imagen del país, y esta debe demostrar que las ciudades, pueblos y regiones del mismo están llenas de personas deseosas de satisfacer sus necesidades. En la industria turística, el cliente es global y es el rey. Se otorga este título no por los derechos heredados, sino porque los clientes tienen la habilidad de engrandecer o dañar su carrera a través de las decisiones de compra que realicen y de los comentarios positivos o negativos que hagan a terceros. (Kotler, Bowen, Makens, Garcia, \& Flores, 2011). Por lo que es preciso transmitir siempre la buena voluntad a los turistas cuando se les está prestando un servicio, pues se necesita que la ciudad y el país logren buena fama, como destinos turísticos.

El término Marca País, o country Brand, nace de la necesidad de los sectores empresariales, y los gobiernos por generar una identidad propia frente a los mercados internacionales. La estrategia de posicionamiento de un país en particular se enmarca en el objetivo de capitalizar el origen de los productos, las empresas y las personas en los mercados globales. La Marca País refleja una visión holística de la imagen de un territorio específico, que debe ser reforzada y enriquecida permanentemente por la inversión en comunicación del país de origen, hacia el resto del mundo (Echeverri, Rosker y Restrepo, 2010).

Se considera que la terminología Marca País, es una estrategia que muchos empresarios y gobiernos tratan de establecer una identidad propia en atesorar el origen de todo el conjunto de elementos del entorno, que comprende cada país en los mercados globales con el fin de llevar a cabo el desarrollo de procesos adecuados de para poder posicionarse o realizar actividades, comerciales y no comerciales (inversionista, visitante, residente) lo percibe como algo diferente a los demás países, que le otorga superioridad sobre los demás, permitiéndole de esta manera obtener una ventaja inicial al momento de realizar la oferta de su producción (Barrientos, 2014). Por lo tanto podría decirse que la Marca País es un factor crucial y decisivo a la hora de posicionar, y mejorar la percepción que se tiene de los servicios, bienes y productos que una nación produce y desea vender en el mercado internacional, ya que le otorga la posibilidad a todos los bienes que son exportados de esa nación en particular, de gozar de todas las ventajas y características positivas que se asocian con ese país o región, permitiéndoles vender más y obtener mejores ingresos a las empresas exportadoras de bienes o servicios. 
Impacto turístico que genera la Marca País "Ecuador ama la vida”

Ecuador no es el primer país en adoptar una marca para sí mismo y se ha unido a la tendencia mundial, que impulsa a las naciones a vender sus identidades con un objetivo claro de reconocimiento y diferenciación que les permita obtener una ventaja competitiva. Es así, que se promueve el posicionamiento de la imagen de Marca País a los posibles compradores del producto Ecuador que se compone de cuatro mundos: Galápagos, Costa, Andes; y Amazonía (Chicaiza, 2014).

Por ello, con el fin de promover la Marca País "Ecuador ama la vida", e impulsar el sector turístico del producto Ecuador se implementaron estrategias de promoción, la cual alcanzó un gran impacto puesto que fortaleció el turismo nacional e internacional.

En Ecuador la Marca País está dando sus primeros pasos, afianzándose primeramente en el turismo, prueba de ello es la última campaña "All you need is Ecuador", enfocada en el turismo receptor. De igual forma en enero del año 2014 se lanzó la campaña "Viaja primero Ecuador", enfocada en el turismo interno y cuyo objetivo es disminuir el porcentaje de divisas, que por turismo emisor salen del país, el momento que el turista nacional viaja al extranjero (Chicaiza, 2014).

Al ritmo del clásico tema musical "All You Need Is Love" de la famosa banda inglesa The Beatles, licenciada por el Ministerio de Turismo; además se realizó la promoción de campaña promocional para consolidar a Ecuador, como un destino de clase mundial, ya que 19 ciudades del mundo, 7 de ellas de Ecuador, fueron testigos del impulso del producto Ecuador como potencial turístico.

La Marca País Ecuador es el primer resultado del Plan Integral de Marketing Turístico, que se fijó como meta doblar hasta el 2006 el número de turistas internacionales (llegar a 1 millón 500 mil turistas), triplicar el ingreso turístico a 1 millón 290 mil dólares y generar 50.000 puestos de trabajo. Para ello, se cuenta con el Fondo de Promoción Turística creado por el Ministerio de Turismo en 2002, cuyo Comité Técnico recomendó realizar una campaña nacional de posicionamiento de la Marca Turística en prensa, televisión y vallas publicitarias (Iglesias y Molina, 2008).

Se considera que según los análisis debidamente realizados por el Plan Integral de Marketing turístico de la Marca País Ecuador, arroja datos cuantificables para determinar el número de turistas que visitan el Ecuador de diferentes países, en un periodo de tiempo con la finalidad que estos se incrementen y en efecto se incremente el rendimiento económico según las metas establecidas, generando más oportunidades de trabajo y así contar con un fondo de promoción. 
Impacto turístico que genera la Marca País "Ecuador ama la vida"

Se considera Patrimonio Cultural a aquella herencia que ha transcendido de generación en generación, y que en sigue viva en la actualidad. Ecuador es una muestra viva de la gran cultura y geografía que lo hace ser merecedor de grandes reconocimientos por su inmensa riqueza cultural y natural.

"El Patrimonio Cultural Material es la herencia cultural propia del pasado de una comunidad que poseen un especial interés histórico, artístico, arquitectónico, urbano, arqueológico" (Ministerio de Cultura y Patrimonio, 2014).

En un sentido más amplio, aunque cultura y patrimonio cultural sean considerados como sinónimos por tener ciertas similitudes, la realidad es que los recursos utilizados por cada uno varían de acuerdo con el contexto. El patrimonio cultural es considerado como acción deliberada y agradable que permite a los turistas disfrutar de los placeres de la vida a medida que esta cambia.

Como menciona Martín "En un sentido más estrictamente antropológico, el patrimonio cultural es un elemento contenido en la cultura, que a su vez contiene otros elementos (...). La herencia nos conecta con la historia, con lo que se transmite de generación en generación. El patrimonio nos vincula al pasado" (Martin, 2003)

"El Patrimonio Cultural Material es la herencia cultural propia del pasado de una comunidad que poseen un especial interés histórico, artístico, arquitectónico, urbano, arqueológico" (Ministerio de Cultura y Patrimonio, 2014).

Ecuador es un país que cuenta con un gran atractivo turístico; a pesar de ser un país considerado relativamente pequeño, ha logrado obtener reconocimientos por la UNESCO al ser nombrado Patrimonio Mundial de la Humanidad. "Ecuador es un país muy rico en patrimonio, tanto cultural como natural. La riqueza patrimonial que poseemos es tan importante que nuestro país ostenta seis importantes reconocimientos concedidos por la UNESCO" (Instituto Nacional de Patrimonio Cultural, 2014).

Lugares como Quito, Cuenca, Galápagos, Parque Nacional Sangay, Pueblo Zápara y Qhapaq Ñan Sistema Vial Andino, son los sitios que han sido considerados Patrimonio Mundial de la Humanidad, en vista de que se ha encontrado la necesidad de conservar y preservar dichos sitios que se destacan por su importancia cultural y nacional. 
Impacto turístico que genera la Marca País "Ecuador ama la vida"

En los últimos años Ecuador ha encaminado sus esfuerzos a convertirse en potencia turística a nivel internacional, su diversidad étnica, cultural, gastronómica y natural, han sido fundamentales para alcanzar dicho objetivo por lo cual el desarrollo de distintas actividades que aporten a la recuperación y conservación del patrimonio natural y cultural son de significante aporte para mantener la riqueza del país.

Se puede considerar que la cultura, es una serie de conocimientos que, en base a recursos adquiridos, por inercia ayudan a mejorar la amplitud de los diferentes cambios de vida, muy a diferencia del patrimonio que intervienen otros recursos.

"El turismo sostenible hace referencia a una forma de turismo que busca la satisfacción de las necesidades actuales de los turistas, la industria turística y las comunidades locales, sin comprometer la capacidad de satisfacer las necesidades de las generaciones futuras; no existe sostenibilidad si no viene dada de una manera integral, teniendo en cuenta muy diferentes aspectos y agentes implicados" (Orgaz, 2013).

"La incorporación de la sustentabilidad en los paradigmas de desarrollo, es un asunto de esencial importancia para la supervivencia de la humanidad, aunado a que se deben conjugar los elementos ecológicos, social con una administración eficiente de los recursos naturales, los cuales explotamos en la búsqueda de un desarrollo económico, en este caso las políticas deben preocuparse en cuanto utilizar ahora y cuanto para las generaciones futuras" (Contreras \& Aguilar, 2012).

Según estos autores, es importante establecer una buena gestión y organización al momento de ofrecer un servicio turístico al público, se debe considerar que un turismo sostenible puede llegar a posicionarse como un mercado de oportunidades, por esto es por lo que se debe tomar en cuenta los siguientes aspectos: social, ecológico y económico como pilares fundamentales del turismo sostenible.

El desarrollo regional, uno de los grandes retos de cualquier proyecto de desarrollo turístico, en diferentes sectores productivos en un mismo marco territorial para crear sinergias entre todos los componentes y sectores económicos, lo cual en muchas ocasiones choca de frente con la desestructuración de gran parte de los espacios turísticos o potencialmente turísticos, especialmente en el interior. Conviene no perder de vista que el turismo no es un motor de desarrollo aislado, sino más bien un sector que se apoya en el marco general de desarrollo de un territorio y una sociedad, 


\section{Impacto turístico que genera la Marca País "Ecuador ama la vida"}

para integrar y aprovechar oportunidades, estructuras y recursos desde los que construir nuevas realidades productivas.

Se debe de considerar que dentro del desarrollo regional es importante que haya una concordancia entre las actividades de cada sector económico, que se pueda analizar cada elemento como el territorio y sociedad, empleando bien las oportunidades que se realicen en un mismo marco territorial para que el proyecto turístico que se desarrolle alcance el éxito deseado.

La estrategia de Marca País, se replica en la generación de una mayor confianza interna y mejor desempeño externo del país. Según Kotler \& Keller (2006) la imagen de marca hace referencia a las propiedades extrínsecas del producto o servicio, incluidas en la forma en que se intenta que la marca satisfaga las necesidades sociales y psicológicas del consumidor. La imagen de marca busca vincularse a las emociones del consumidor (Kotler, Setiawan \& Kartajaya, 2010).

La finalidad de una Marca País, es alcanzar y mantener el posicionamiento. El posicionamiento es con frecuencia una acción crucial y estratégica para una empresa, un producto o un país, porque centra su atención en ocupar un lugar central en las percepciones y decisiones de su mercado objetivo (HarrisonWalker, 2011). La imagen de una Marca País se construye sobre la realidad del país, el mensaje que se comunica y la influencia de hechos históricos más destacados (Bertrán Vall, 2003).

Se considera que actualmente los países compiten para atraer el turismo, y ser el más atractivo a nivel mundial y estratégicamente se emplea la Marca País, permitiendo la capacidad a cada región distinguirse y darse a conocer a nivel mundial, obteniendo una ventaja como el de posicionarse y obtener un reconocimiento internacionalmente.

La campaña publicitaria 'All You Need Is Ecuador' es parte de las estrategias de promoción turística del país, que ha sido difundida desde el mes de abril del año 2014 y que ha tomado importancia a nivel mundial por su rápida difusión en los medios de comunicación. Esta campaña tiene como principal objetivo el incentivo del turismo receptor y posicionar al país como un destino turístico para conocer. El Ministerio de Turismo ha establecido como meta lograr un incremento del 10\% anual en la llegada de extranjeros en el 2015, acorde con estimaciones del gobierno, la llegada de extranjeros alcanzaría 1'560.429 personas (Ministerio de Turismo, 2015). 
Impacto turístico que genera la Marca País "Ecuador ama la vida"

Dado que esta campaña publicitaria se ha dada a conocer en distintos medios de comunicación a nivel nacional e internacional, se puede observar el impacto que tiene la misma en cada uno de los turistas que visitan las tierras ecuatorianas, donde se establece que lo esencial es el incentivar el turismo y posicionar al Ecuador como un destino turístico para conocer.

Es importante recalcar los mejores destinos turísticos del Ecuador \& Galápagos, la cual se puede visualizar en la tabla 1.

Tabla 1 Los mejores destinos del Ecuador \& Galápagos.

Mejores destinos

1. Ciudad de Cuenca (Patrimonio Cultural de la Humanidadde la UNESCO).

2. Ciudad de Guayaquil - Malecón 2000

3. Ciudad de Quito (Patrimonio Cultural de la Humanidad de la UNESCO)

4. Volcán y Parque Nacional Cotopaxi

5. Bosque nublado de Mindo

6. Pueblo de Montañita y sus playas

7. Playa de Salinas en Santa Elena

8. Ruta de Spondylus

9. Pueblo de Riobamba y Volcán Chimborazo

10. Pueblo de Puyo - Actividades en la selva amazónica

11. Ciudad de Manta y Parque Nacional Machalilla

12. Mercado de Otavalo - Actividades en el Lago San Pablo

13. Línea Equinoccial - Monumento en la Mitad del Mundo

14. Playa de los Frailes en Manabí

15. Ciudad de Loja - Vilcabamba

16. Laguna volcánica del Quilotoa

17. Playa de Canoa en Manabí

18. Reserva Faunística Cuyabeno en Sucumbíos

19. Pueblo de Misahuallí - Actividades en la selva amazónica

20. Ciudad de Ambato - Mercado de Saquisilí

21. Ruinas incas de Ingapirca

22. Playa de Mompiche

23. Playa de Puerto López en Manabí

24. Laguna de Cuicocha - Reserva Ecológica Cotacachi Cayapas

25. Parque Nacional Cajas

26. Termas de Papallacta - Reserva Ecológica Antisana

27. Isla de la Plata - Parque Nacional Machalilla en Manabí

28. Ferrocarril de Ibarra a Salinas

29. Parque Nacional Yazuní 
Impacto turístico que genera la Marca País "Ecuador ama la vida"

30. Bahía de Caráquez - Isla Corazón

31. Avenida de los Volcanes

32. Ciudad de Baños de Agua Santa - Cascada Pailón del Diablo

33. Lagunas de Mojanda - Pesca y excursionismo

34. Cascada de Peguche - Observación de aves y acampadas

35. Laguna de Yahuarcocha

36. Reserva Ecológica El Ángel

37. Zaruma - Minas de oro, arquitectura y café local

38. Bosque petrificado de Puyango

39. Playa de Crucita - Parapente y avistamiento de ballenas

40. Ferrocarril Nariz del Diablo - Alausí

41. Playa El Murciélago

42. Playa de Pedernales

43. Playa de Olón

44. Cuevas de Jumandí

45. Cascada de San Rafael - Volcán Reventador

46. Playa de Puerto Cayo - Surf

47. Termas de Chachimbiro en Urcuquí

48. Cascadas del Río Pita - senderismo

49. Ayangue - Buceo

50. Pueblo y volcán Cayambe

51. Ciudad de Tulcán

52. Parque Nacional Podocarpus

53. Isla de Muisne

54. Salinas, Ballenita, Olona y Montañita - Surf

55. Cascada Manto de la Novia

56. Pueblo y volcán Cotacachi

57. Río Napo

58. Playa Rosada

59. Ciudad de Macas

60. Carnaval de Guaranda - Bosque Protector Cashca Totoras

61. Reserva Ecológica Los Illinizas

62. Ciudad de Machala

63. Refugio de Vida Silvestre Pasocha

64. Salinas de Bolívar

65. Reserva Ecológica Limoncocha

66. Lagunas de Ozogoche

67. Cráter del Pululahua

68. Santo Domingo de los Tsáchilas

69. Parque Nacional Sangay

70. Jama Coaque

71. Parque Nacional Llanganates

72. Lagunas de Imbabura

73. Lagunas de Atillo

74. Chordeleg

75. Ayambe - Buceo y avistamiento de ballenas 
Impacto turístico que genera la Marca País "Ecuador ama la vida"

76. Playa de San Vicente

77. Comunidad indígena de Saraguro

78. Cascadas de Girón

79. Playa de Punta Blanca

80. Volcán Pichincha

81. Playa de Santa Marianita - Surf de vela

82. Gruta de la Paz

83. Parque Histórico de Guayaquil

84. Reserva Ecológica Cayambe Coca - Termas de Oyacachi

85. Volcán Tungurahua

86. Camino del Inca (Qhapaq Nan)

87. Teleférico de Quito

88. Parque Nacional y Reserva Marina de Galápagos

89. Ciudad de Tena

90. Volcán y Reserva Ecológica Chimborazo

91. Reserva Ecológica Manglares Churute

92. Parque Itchimbía in Quito

93. Bosque nublado de Intag

94. Ciudad de Ambato - Carnaval de las Frutas y las Flores

95. Complejo Turístico Casa Blanca

96. Ciudad de Ibarra

97. Valle del Chota y Comunidad Afro ecuatoriana

98. Playa de Atacames

Fuente: (http://ecuadoramalavida.blogspot.com/, 2011)

\section{Conclusiones}

El principal objetivo de una Marca País, es poseer una reputación de productos de alta calidad, deseos por visitar o realizar una formación académica y sobre todo las percepciones de buena infraestructura.

Tener una marca país fuerte, conlleva a poseer una ventaja competitiva cuantificable en relación con las visitas de turistas, inversión, así como la preferencia de los consumidores hacia los bienes o servicios ofertados por los países.

La fuerza de la percepción de una Marca País se debe a la economía, política, tecnología, innovación y el ambiente, quienes comunican percepción de excelente reputación hacia el presente y futuro.

El análisis permitió visualizar un panorama general de la conceptualización y recopilación de información de "Marca País", la cual podemos reflexionar que existe una mala imagen sobre nuestra Marca País en el exterior, dado que no se transmite confianza y seguridad; es importante explotar las 
fortalezas que posee Ecuador, para así obtener inicialmente un compromiso de la nación y demostrar todos los potenciales turísticos que posee nuestro hermoso país, al mundo entero.

\section{Referencias Bibliográficas}

Amaral Moretti, S. L., \& Lenzi, F. (2013). Superando la estacionalidad turística. Dialnet, 3-5.

Barrientos, P. (2014). El desarrollo de la marca país. Revista Finanzas y Política Económica, pág. 2.

Bejarano, N. (2012). Planificación Turística. UTEG, 14-16.

Bernal, C. (2006). Metodología de la investigación: para administración, economía, humanidades y ciencias sociales. Naucalpan, Edo. de México: Publicaciones Pearson Educación.

Bigne, E., Font, X., \& Andreu, L. (2014). Marketing de Destinos Turísticos. Madrid: Esic.

Caimi, M. (2003). DISCURSO DEL MÉTODO: Volúmen 39 of (Clásicos del pensamiento) Colihue Clásica. Argentina: Ediciones Colihue SRL.

Cantallops, A. S. (2013). Marketing Turístico. Madrid: Hispana.

Castellanos, M. (2011). El turismo en un Ecuador. Revista Gestión, 2.

Chicaiza, L. y. (2014). La marca país: su origen y evolución, caso Ecuador.

Costa, J., \& G. Bosovsky. (2012). Los 5 pilares del branding: Marca País. Costa Punto ComEditor, Tiana, Barcelona.

http://ecuadoramalavida.blogspot.com/. (2011). http://ecuadoramalavida.blogspot.com/. Obtenido de http://ecuadoramalavida.blogspot.com/

Instituto Nacional de Patrimonio Cultural. (2014).

Keller, K. (2012). Administración estratégica de marca. México: Pearson Education.

Kotler, p., Bowen, J., Makens, J., Garcia, J., \& Flores, J. (2011). Marketing Turístico. Madrid: Pearson. 
Impacto turístico que genera la Marca País "Ecuador ama la vida"

Martin. (2003). Nuevos turistas en busca de un nuevo producto, el patrimonio cultura. Pasos: revista de turismo y patrimonio cultural, Vol. $1 \mathrm{~N}^{\circ} 2$, pág. $155-160$.

Marzano, G. (2013). Marca País: Un enfoque metodológico. IDE Business School, USFQ.

Ministerio de Cultura y Patrimonio. (2014).

Ministerio de Turismo del Ecuador. (2012). Evaluación marca turística, percepción, hábitos y preferencias del turista no residente que ingresa al Ecuador. Quito: PROPRAXIS.

Ministerio de Turismo del Ecuador. (2013). Estudio de tendias del turismo no residente en el Ecuador. Quito: GKF.

Ronquillo, S. C. S. (2016). La difusión de la campaña "All you need is Ecuador" y su impacto en el turismo receptivo. Revista Empresarial, 10(3), 56-65. 